\title{
Energy-Aware Load Direction for Servers: A Feasibility Study
}

\author{
Shane Case, Furat Afram, Erdem Aktas and Kanad Ghose \\ Department of Computer Science \\ State University of New York, Binghamton, NY 13902, USA \\ \{shane,fafram1,eaktas,ghose\}@cs.binghamton.edu
}

\begin{abstract}
We address the problem of improving the energy efficiency of servers that provide web-based services, including services provided through clouds. We propose an automated technique for allocating workload to servers to operate the fewest number of servers that are needed to cope with the instantaneous workload, leaving some headroom for workload surges. The technique requires no a priori knowledge about individual workloads and manages the server states explicitly. We use synthetic scripts, a small server setup and actual energy consumption measurements to show that the proposed system achieves non-trivial energy savings at typical operating regions of traditional server configurations.
\end{abstract}

\section{Introduction}

In recent years there has been an increased demand for web based applications such as media streaming, search services, e-commerce, email, social networking, and cloud computing. The growth in these services has been fuelled in part with the widespread use of web-enabled mobile devices. Such unprecedented growth in the demand for these services has spurred a growth in the number and capacities of data centers worldwide. Data centers are a growing source of the total national energy consumption: based on 2005 data [1], the total energy consumption of all U.S. data centers alone was about 1.5 percent of the U.S. energy consumption. Since 2005, the energy consumed by data centers has also grown annually in double digit percentages, demanding the need to design and operate data centers in an energy efficient manner.

This paper addresses a central issue in reducing the energy consumption of data centers. We propose and evaluate a technique to operate servers in an energy-efficient manner. The proposed scheme recognizes the low energy efficiency of modern servers at light load levels [2, 3] and operates the fewest number of servers that are needed to cope with the current load level. The active servers are operated at a high loading level to realize a high degree of energy efficiency (as measured by performance per Joule of energy spent). Inactive servers are maintained in the deep sleep mode whenever possible to reduce energy wastage from idling. Current system-level load trends are used to turn servers on in advance to deal with increase in load levels. Energy measurements from a prototype implementation demonstrate that significant energy savings can be realized over a baseline load directing scheme within typical operating regions of a traditional server installation.

The technique proposed and evaluated here has the following characteristics:

- Energy-Aware Load Direction: It uses realistic, direct measures of server loading for directing the offered workload to servers.

- Request-Oblivious Redirection: It performs load direction without any a priori knowledge of the individual requests.

- Simplicity: It uses a simple control mechanism to steer workload towards active servers and to activate/deactivate servers in anticipation of an increase or reduction in the offered workload.

- Independence: The load direction scheme permits each server to use any local energy management scheme as they see fit.

Figure 1 depicts the typical situation in a modern server by plotting the server power dissipation (red line) and the energy efficiency (green line, measured as performance realized per Joule of energy expended) against the server load. A great part of this inefficiency is due to the relatively high server power dissipation at zero load (also called idle power, shown as P0). The high noload power dissipation P0 is due to several factors, 
including inefficiencies in the various power supplies/converters, dissipation in server semiconductor components at no load (including leakage power dissipation), high power dissipation in disk drives that are not being used but spinning, and use of overrated power supplies and components. Unless server component designs are radically changed, it is unlikely that P0 can be reduced dramatically [4].

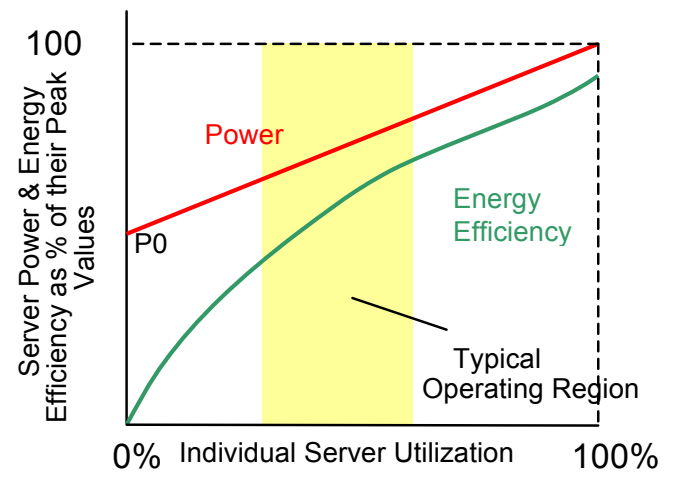

Figure 1. Server power dissipation and energy efficiency as a function of the offered load.

From an energy-efficiency standpoint, the impact of relatively high idle power dissipation in servers is exacerbated by the fact that typical server installations are overprovisioned. That is, servers are rarely operated at or near their peak loading region which these installations are designed to handle. Servers typically operate at low load levels - typically at $30 \%$ to $40 \%$ of their maximum capacity [3]. The wide deployment of virtualization technologies has made it possible to consolidate the offered workload onto a few servers to operate these servers at a high load region and thus realize high energy efficiency. At the same time, the idle servers are kept in a deep sleep state to conserve energy. Unfortunately, load consolidation using virtualization mechanisms is not an automated process and is instead under the full control of human operators, who use tools like $[5,6]$ to examine the current workload and/or use prior knowledge of workload arrival patterns to determine (or define rules that activate) the appropriate number of on-line servers. These operators then manually deploy the necessary load redirection and virtual machine migration mechanisms to consolidate load on the active servers. Operators tend to overprovision the number of active servers to handle load, consequently the full benefit of virtualization is not realized.

It is thus desirable to have an energy-aware load direction technique that is automated, making

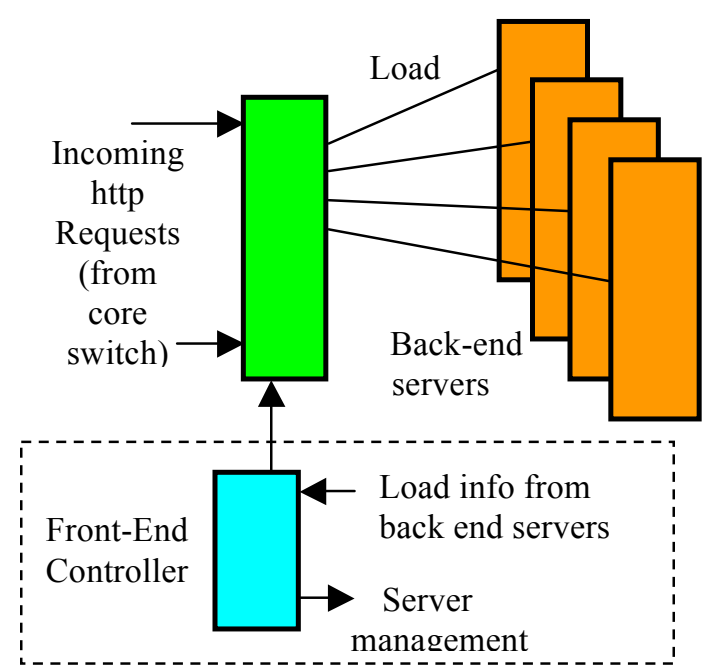

(a) Targeted system; additional components for the evaluation svstem are shown within the dotted box

\section{Figure 2. Targeted server architecture and evaluation system details}

use of actual fine-grained load information from the active servers and is oblivious to the individual server requests. In this paper, we propose and experimentally evaluate a load direction technique that actually has these desirable features.

\section{Related Work}

With the dramatic increase of web based applications and services in the recent years, many techniques have been proposed for directing incoming load to servers and to manage servers in a manner that reduces the overall energy consumption. The technique presented in this paper has some superficial resemblance to the work of Rusu et al [7], where load direction was performed for requests to static and dynamic pages, based on the number of pending requests and on the power dissipation estimates of the requested tasks. The frequency-voltage setting of the server machines were also controlled explicitly by the load director facility. In contrast to the work of Rusu et al, our solution takes a more direct approach at energy-aware load direction:

- We make use of the actual current loading on each server, not the number of requests.

- Our technique requires no a priori knowledge of the tasks or their power dissipations at different frequency-voltage settings.

- Our mechanism does not require packetspecific information for its decision process, 
making it possible to deploy our mechanism with existing load balancing switches.

- Our solution permits the server-local speedstep governors to adjust the DVFS settings.

- We evaluate our system on the basis of energy consumption; Rusu et al uses the power dissipation.

A load direction technique called NapSAC was introduced in [8]. NapSAC targets a power aware cluster based on three classes of machines (server, mobile and embedded), determining the class based on the number of requests. The authors calibrated the design based on maximum number of requests that each class can handle and use a knapsack algorithm to run each server at or near optimal utilization. The decision process of NapSAC also requires information from individual incoming packets and thus has some of the limitations of Rusu et al's techniques, as mentioned earlier.

In [9] Lang et al present a method for saving the energy consumption of servers which shuts down unnecessary servers based on the total system utilization with the support of data replication. While the paper gives details about how to replicate the data for future availability and select the victim server to shutdown, it does not give enough explanation about how the system and individual server utilization are calculated.

In [10] Alspaugh et al propose an approach to reduce server energy consumption by putting the unnecessary servers to the S3 sleep state based on the overall system utilization, with system utilization represented indirectly by the number of concurrent requests that are being served. It is unclear how the system performs on dynamic jobs/workloads, as system utilization for such workloads is not represented by the number of requests served.

In [11], the authors introduce a hybrid data center design that mixes low power servers and high performance servers to save energy. The technique does not have a centralized management node and scalability issues as a function of the degree of heterogeneity in the server pool are not discussed. The technique of [12] also follows the method of waking up servers based on an incoming request(s). However, this scheme requires hardware changes to realize power savings. Our scheme differs from the technique in that we require no hardware modifications, and the allocation of requests to servers is based on the load placed on each server, rather than indirect indicators of the server loads.

In [13], Pinhiero et al discuss a load balancing and server activation/shutdown mechanism which consolidates a load on a few servers and turns off the remaining servers, implemented within a customized cluster OS. The fairly complex control theoretic management approach relies on the customized cluster/server OSs to collect resource usage information. This information is then used to avoid performance degradation when servers are being shut down. Tasks are also migrated to facilitate a shutdown decision. This technique is thus not applicable to non-clustered designs and in particular the typical server architecture that we target. In [14], Verma et al discuss scheme of migrating VMs to the minimal number of servers and sending unused servers to a low power state. Their load gathering method requires the IBM Active Energy Manager APIs and VMM support for migration; our scheme requires no migration methods and gathers load information directly from programmable MSRs.

\section{Load and server management}

Our proposed automated load balancing and server management system requires a control processor, as shown in Figure 2. The server management system, called the Front-End Controller (FEC) collects actual loading data from the servers and uses this information to either activate standby servers or to take servers off the list of active servers and eventually put them into the low power suspend-to-RAM mode. For the feasibility study of our proposed scheme, we use a hardware high-performance load balancer. The control processor itself can be a simple embedded system, as our management technique is very simple.

In our system, each server runs a daemon called the Back-End daemon (BED) to report load information to the FEC and to request a transition to the standby mode when it has finished all of the jobs allocated to it. Responses to the FEC from the BED also serve as heartbeats for the back-end server (BES) on which it runs. The BED uses some OS data structures and the hardware instrumentation counters to report the actual server load, instead of the system reported load average, which is only reported every 5 seconds. They are implemented as a stand-alone kernel module and can be easily ported to any OS. No direct modifications of the server kernel are thus needed in our scheme.

The Front End Controller (FEC) is the core component of the proposed energy smart load balancer. It communicates with all the BEDs, the hardware load balancer and controls which 
server(s), if any, go to standby or need to be activated, after determining the number of servers necessary for handling the incoming load.

The FEC can work with any dynamically configured load balancer, including all but the very low-end load balancing switches. There are no

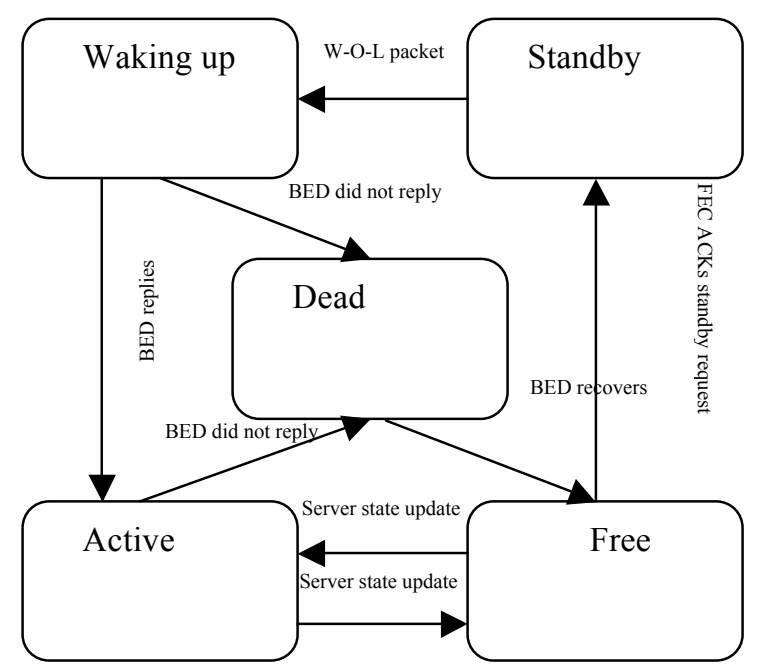

Figure 3. Server states

modifications to the load balancer, and the FEC is a completely independent process running on the control processor. The only change required to FEC would be the interface with a particular load balancing suite. In this case, the load balancer can be reconfigured dynamically via its control interface to mark a server as "down in maintenance mode" or "up" in the active mode.

The load balancing mode and identities of the active servers used for serving the incoming requests by the load balancer can be controlled via a SOAP interface to make online configuration changes. Communications with the back end clients is done by using network sockets. The FEC uses two pairs of UDP sockets to communicate with a BED instance, one pair to receive the server load metrics sent by the BED, and the other pair to listen to and respond to any request made by the BED to put its associated server in the standby mode.

To provide fine-grained estimation of a server's energy consumption, we rely on the existing architectural performance/instrumentation counters in modern microprocessors [15]. One of the fixed performance counters, referred to as UNHALTED_CORE_CYCLES (UCC), counts the number of cycles for which the processor has been active and not in either HLT or any deeper sleep state. By comparing this counter to the Time Stamp Counter (TSC), which records the elapsed time in $\mathrm{CPU}$ cycles, we quantify the CPU loading at a finer granularity than what is provided by the kernel. Specifically, for an individual server, the ratio of the UCC value to the TSC value indicates the CPU loading. The average system load is considered as the weighted sum of the individual utilization of each server. For the system used for our experimental assessments, the servers are identical (and so the weights are all unity).

Other measures of server loads based on the MSRs and other software counters are possible, accounting for both compute and I/O bound jobs or their combinations but these are not reported in this paper.

In order to read the MSR/counter values, a kernel interface was implemented for use by the BED at each server. This kernel interface will need to activate the performance counter interface by modifying the appropriate model specific registers, and output the counter values to user space. Utilizing the proc file system [4], performance counters can be read by the BED and upon reading these values, the counters are reset. In our implementation, the performance overhead of reading and resetting the counters is less than $1 \%$.

In many modern servers (such as [16]), system level firmware exists to report various metrics that indicate system load and other system level parameters of interest. This information is either available through a standard interface (IPMI) or through a proprietary interface. Such a facility can be used to report load information at a finer grain to our load balancer, but were unavailable on our test platform and thus were not used.

From the FEC's standpoint and based on some of the preceding discussion, a back end server can be in one of the following states:

- Active: the server is enabled.

- Free: The server is disabled in the configuration and the server may still be serving requests previously assigned to it.

- Standby: the server is disabled in the configuration and it is suspended to RAM.

- Waking up: the server is disabled in the configuration and a Wake-On-LAN packet has been sent to it but it is not yet ready to serve requests.

- Dead: BED did not reply after a wake-on-LAN signal was sent to the server, or the server did not reply to load request $n$ consecutive times (where $\mathrm{n}$ is a small number).

Figure 3 depicts the directions of possible transitions among these states. In our prototype system, servers take about 7 seconds to move from the standby state to the active state. 


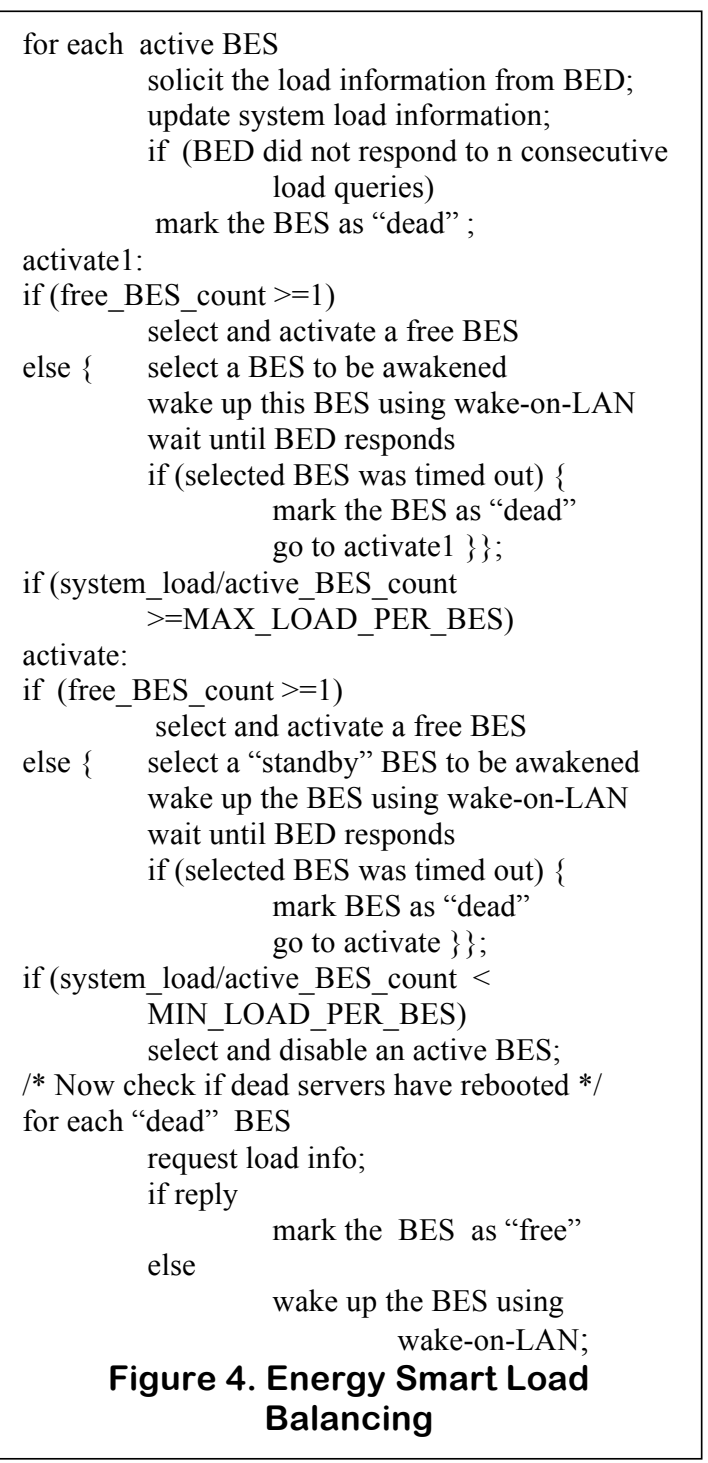

The FEC implements an energy smart load balancing algorithm shown in Figure 4 to decide whether to enable a back-end server (BES), disable, or to continue using the same server configuration. The FEC maintains a local database that is updated with the status of each server on a periodic basis. The algorithm implemented by the FEC relies on the use of two load thresholds, MAX/MIN_LOAD_PER_BES. These two load thresholds are used to activate, deactivate, or suspend servers using the algorithm of Figure 4.

If the FEC receives a standby request from a BED instance, it waits until it finishes calculating the average system load and then checks if there is any need for an extra server to handle the load. If the load is below the MAX LOAD PER BES, the FEC allows requesting server to suspend. To avoid sending multiple servers to sleep, the FEC allows only one server to sleep every time it calculates the system load.

The values of the load thresholds used by the FEC have to be determined through calibration to give a good balance between the energy savings and performance loss. When calibrating these values, the system administrator has to keep in mind that the average load will have to be scaled when the number of active servers changes. For example if the average load per server is $90 \%$ divided between three servers, after enabling a fourth server, if the load stayed the same, the average load per server would be $67.5 \%$. If the system administrator fails to scale the average server loads on activating new servers (that is, chosen 90 and 67.5 as thresholds) the FEC will disable and enable servers at the end of every load reporting cycle when the steady load hovers around the thresholds. The equation below gives the number of servers when this can happen; whenever $N_{\text {servers }}$ is an integer, the front end controller will be turning on or turning off client servers at the end of each load calculation interval:

$$
\mathrm{N}_{\text {servers }}=\text { MIN_LOAD/(MAX_LOAD }- \text { MIN_LOAD) }
$$

Note that we have assumed identical backend servers for simplifying the exposition of our energy smart load balancer. Some obvious generalizations are possible when the servers are not identical, including a choice in selecting a server that has to be turned on or turned off.

\section{Experimental validation}

To evaluate our energy smart load balancer, we utilized four Dell Precision R5400 servers as our BESs, each with 2 quad core Xeon E5450 CPUs and 16 GB RAM as our back end servers (BES). Each BES has a power dissipation of $155 \mathrm{~W}$ when on and idle, and $10 \mathrm{~W}$ when in the suspend-to-RAM state. In our setup, a server takes up to 7 seconds to return from suspended to the active state. An F5 Big IP 3900 load balancing switch is used to balance web traffic, which is connected with a Cisco Catalyst 2590 fast Ethernet switch, with ports configured as PortFast ports, to minimize latency created by spanning tree configuration. This switch also connects the FEC to the BESs. Power and Energy dissipation measurements were taken with a Summit Powersight PS 2500 power meter with data logging capability. Independent client machines were used to generate the requests. The low and high thresholds for each BES were set at $30 \%$ and $75 \%$, with load values refreshed every second. 
The load generating scripts (our "benchmarks") that we chose to evaluate our load balancers were simple PHP scripts, one performing integer arithmetic for mainly CPU bound operations, a second that creates a bitmap image and compresses it to JPEG, and a third that computes the MD5 digest of several distinct files. Each of these benchmarks was run with the default load balancing setting (weighted round robin), and with load balancing and our energy smart load balancer (ESLB). These scripts will be executed by the ab benchmark [17] provided in the Apache web server source tree. All of the measurements reported are the harmonic mean of 5 sets of runs.

\subsection{Integer Arithmetic}

For the integer arithmetic benchmark, a variety of integer arithmetic operations are performed in a loop for 1 million iterations. In Figure 5 (a), the y axis shows number of requests per second, and the $\mathrm{x}$ axis shows the utilization. For the runs, requests were generated in groups of 5000 and the number of concurrent connections was increased after the issue of all requests from the group before the requests from the next group of 5000 requests were issued to the system. The reason for increasing the number of concurrent connections to the server was to increase the load offered to the server system. This was done for all of the workloads described in this section.

From Figure 5 (a) we can see that no performance overhead is incurred by ESLB compared to the base case, default load balancing. This is primarily due to the fact that the arithmetic scripts are CPU bound, taking full advantage of the 8 cores on each BES. They also have no conflicting resource requirements beyond the CPU. The arithmetic scripts only use resources private to each core and thus two executions of the script will finish at the same time regardless of whether they are executed on the same machine or executed on different machines.

Figure 5 (b) depicts the (measured) average energy expended by each request to execute the arithmetic script on the average, as a function of the overall system utilization. With the arithmetic workload, when the system utilization is less than 19,38 , and 56 percent, we have 1, 2, and 3 active BESs, respectively for our energy smart load balancer (ESLB). The more pronounced peaks in the energy expended per request curve of Figure 5(b) occur when a new BES is activated with increasing load - for a short while the servers do not remain at high utilization level.

\subsection{JPEG Compression}

For the JPEG script, each script generates an $800 \times 600$ bitmap image, superimposes a string to the image, and compresses it to the JPEG format. This leads to a high degree of $\mathrm{I} / \mathrm{O}$ write operations. Jpeg compression has a lot of memory writes, generating contention among requests co-running on a socket. Thus, as seen in Figure 6 (a), at low load levels, the request execution times are prolonged due to contention among the requests in our system (relative to the base case). This is because we have fewer servers (and cores) active at low loads and requests are concentrated on the fewer active cores. When the utilization level reaches about $56 \%$, three BESs are activated and the completion times of the scripts are almost identical to those for the base case.

From the view of energy dissipation, in Figure 6 (b), which depicts the average energy consumed by each request served, our scheme can save energy over the base case up until all servers are powered on. The energy expended per served request also shows spikes at some utilization levels due to contention among the memory operations in the course of creating the image and compressing it to JPEG format. In addition, turn-on spikes similar to those shown in Figure 6(b) are also present.

\subsection{MD5 Checksum}

The MD5 script simulates a workload with a high amount of I/O read operations. Each script reads a large randomly chosen data set from the BES-local disk and generates the MD5 digest for the data. The overall trends of the results shown in Figures 7(a) and 7(b) are similar to those shown in Figure 6. Here again, resource contention causes our scheme to have longer service times at lighter system loading (with fewer servers active). The spikes in Figure 7(b) are also due to turn-ons and memory/disk I/O contention.

\subsection{Hybrid Benchmark}

The Hybrid benchmark is a mix of the three benchmarks described earlier and shows how our system responds to a heterogeneous request mix. Figure 8 shows the number of requests per second at different utilization levels and the energy consumed by each served request. Within each group of 5000 requests, the request scripts were chosen randomly but we ensured that all runs had an identical request generation sequence. In this particular case, the 
request service rates for ESLB and the base case are almost identical, possibly because of the reduction of resource conflicts as a result of randomly interleaving different types of requests.

\subsection{Summary of Energy Savings}

For the workloads studied, energy savings achieved by ESLB over the base case are quite considerable at lighter system load levels and the energy savings continue well over system loads of $50 \%$. At a $30 \%$ system load, the energy savings range from $28 \%$ (jpeg) to $38 \%$ (arithmetic) and at $40 \%$ system load the energy savings range from $14 \%$ (hybrid) to about 19\% (MD5). These are clearly non-trivial energy savings.

\subsection{Impact of Sudden Increases to Load}

With sudden increases in the workload offered to the system, the base case clearly provides the best service times, as it keeps all BESs on, irrespective of the system load. In contrast, our energy-smart load balancing system is momentarily unable to cope with a sudden load surge, as our basic scheme activates servers one at a time with load increases. The results shown in Figure 9 depict the impact of request service time for our scheme as such (ESLB) and the base case. In the scenario shown, the offered load level jumps up from $0 \%$ to $100 \%$ at time $\mathrm{t}=0$ seconds. Figure 9 (a) depicts the request processing rate, while Figure 9 (b) depicts the system power consumption. Because ESLB activates one server at a time, it takes about 11 seconds for ESLB to catch up with the request processing rate for the base case.

There is thus a tradeoff between the energy savings realized at lighter loads (which are the most dominant operating regions) and the service time for requests. To improve the response time for transients, several simple changes can be made to ESLB to improve its response time. One change may be to keep a number of reserve servers on hand.

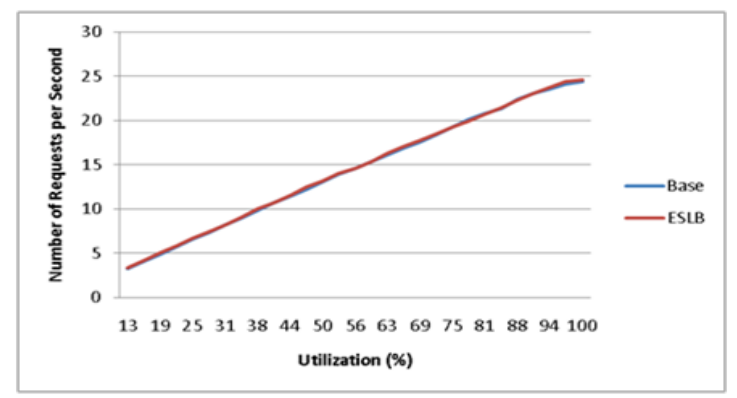

Another will be to activate more than one server at a time. Other variations are possible, including changing the number of servers turned on a time based on the slope at which the load increases.

Combinations of some of these solutions are also possible. Figure 10 (a) depicts a simple extension to ESLB, labeled ESLBm, where two servers are turned on after ESLB senses the load increase.

In this case and as expected, ESLB catches up with the processing rate of the base case earlier than ESLB does in between load levels of $50 \%$ and upwards.

With gradual increases in the offered load, ESLB's request service rates are identical to that of the base case. This is shown in Figure 10 (a), where the offered load has been steadily increased from $0 \%$ to $100 \%$ in 16 steps. The corresponding power dissipations and the overall system utilizations are depicted in Figure 10 (b).

\section{Future work and conclusions}

The current work has clearly demonstrated that non-trivial energy savings are possible at low load levels - the typical and most frequent operating range for real servers - through fully automated load consolidation on a few active servers.

The proposed ESLB technique was demonstrated on a scaled down system but this, by no means, implies that our system is not scalable. Our ongoing work is specifically directed at scaling our solution to larger installations. One way to do this is to implement a multi-level energy-aware load balancing mechanism which views the servers as an ensemble of server groups with a server group consisting of a number of servers. A server group could be a rack of servers or a number of server racks. The servers within a group can be managed using the technique described earlier, with a load balancer as described for directing requests to servers within the group. At the upper level, a global load balancer and server management

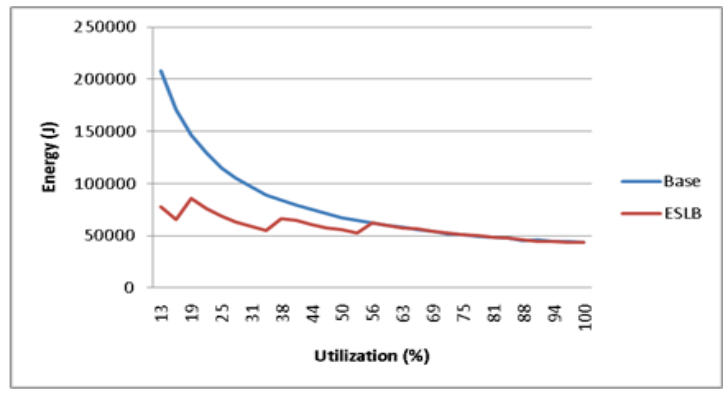

Figure 5. Arithmetic benchmark: (a) requests per second, (b) energy per request 

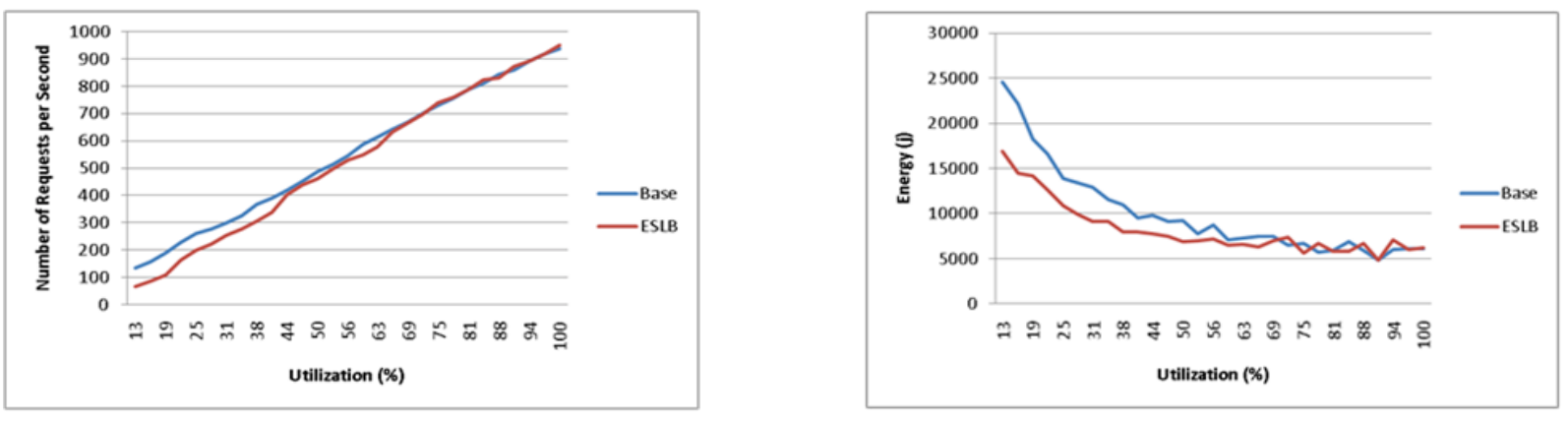

Figure 6. Jpeg benchmark: (a) requests per second, (b) energy per request
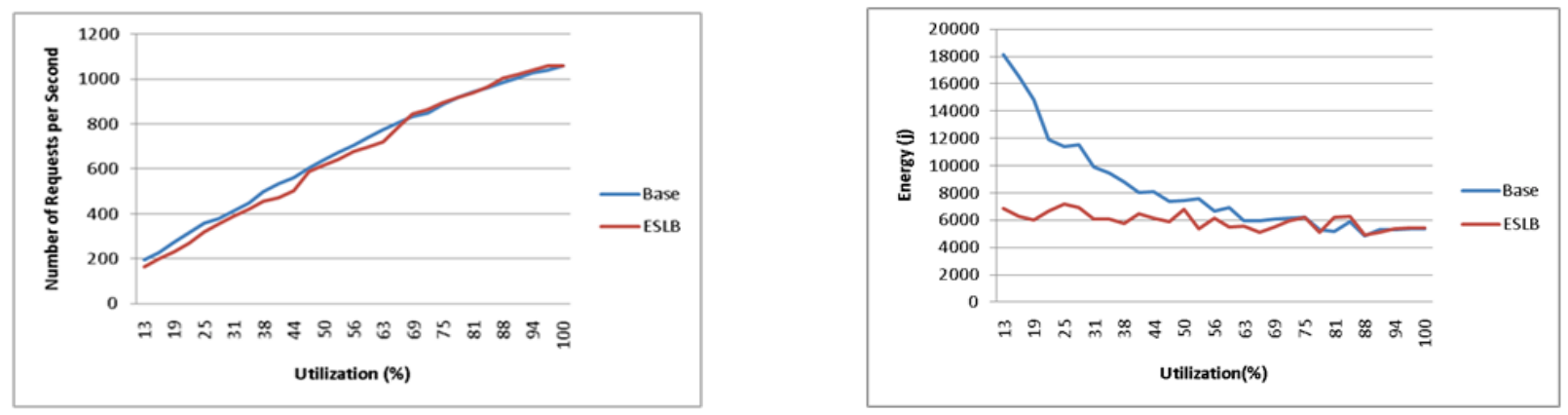

Figure 7. MD5 benchmark: (a) requests per second, (b) energy per request
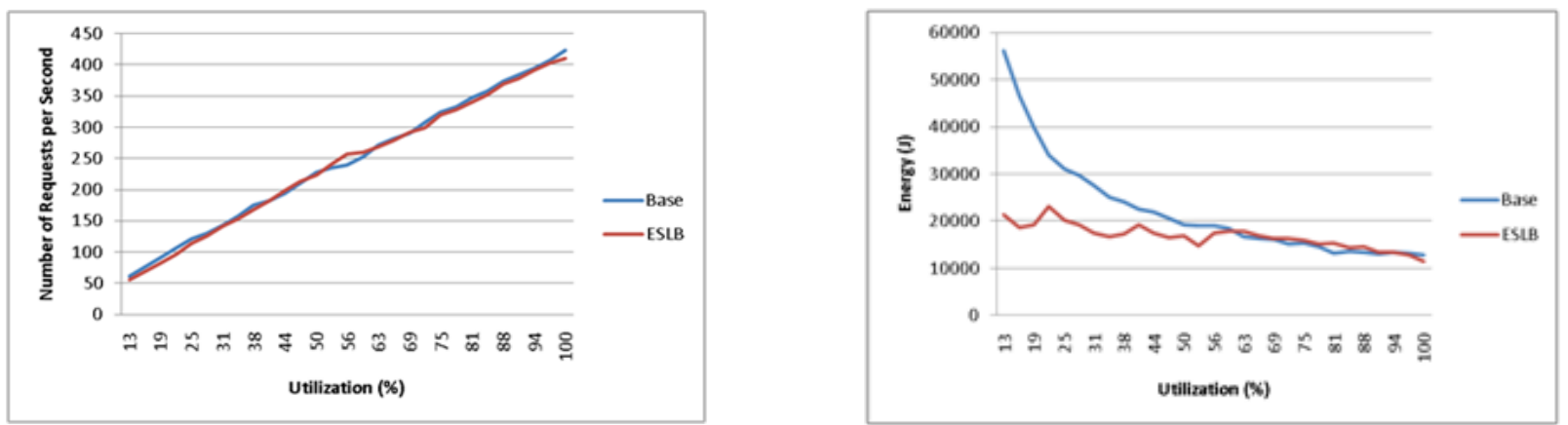

Figure 8. Hybrid benchmark: (a) requests per second, (b) energy per request
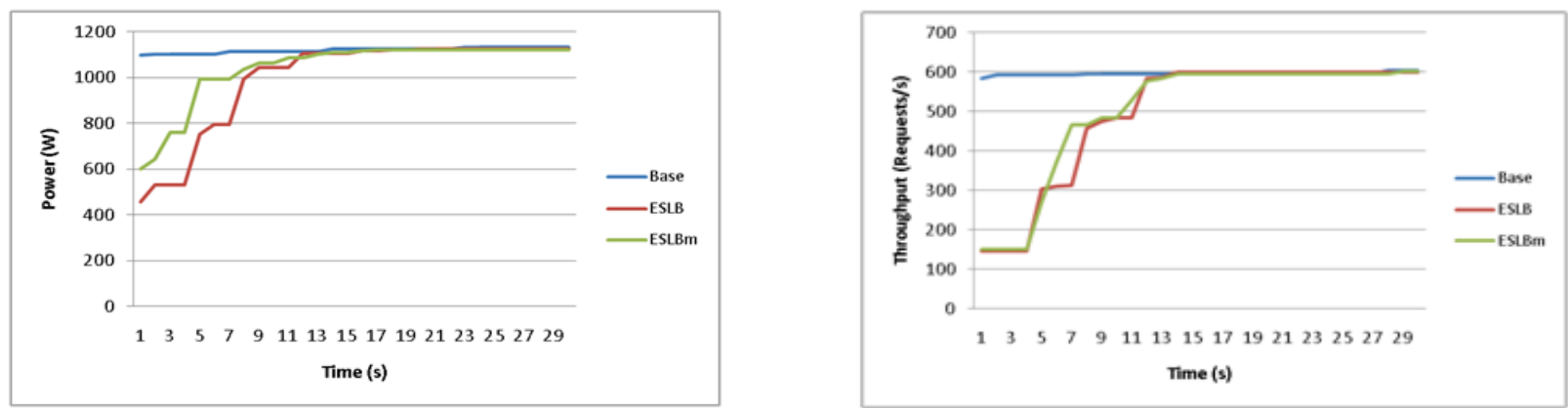

Figure 9. (a) Power, (b) throughput comparisons between the base system and the ESLB scheme when the load dramatically changes from 0 to 100 percent.

mechanism, analogous to the one described earlier can be used to activate or deactivate server groups and to direct load to active groups. It is possible to use simple round-robin load balancing at this level, but additional gains in terms of energy-efficiency and better heat distribution in a server facility can be 

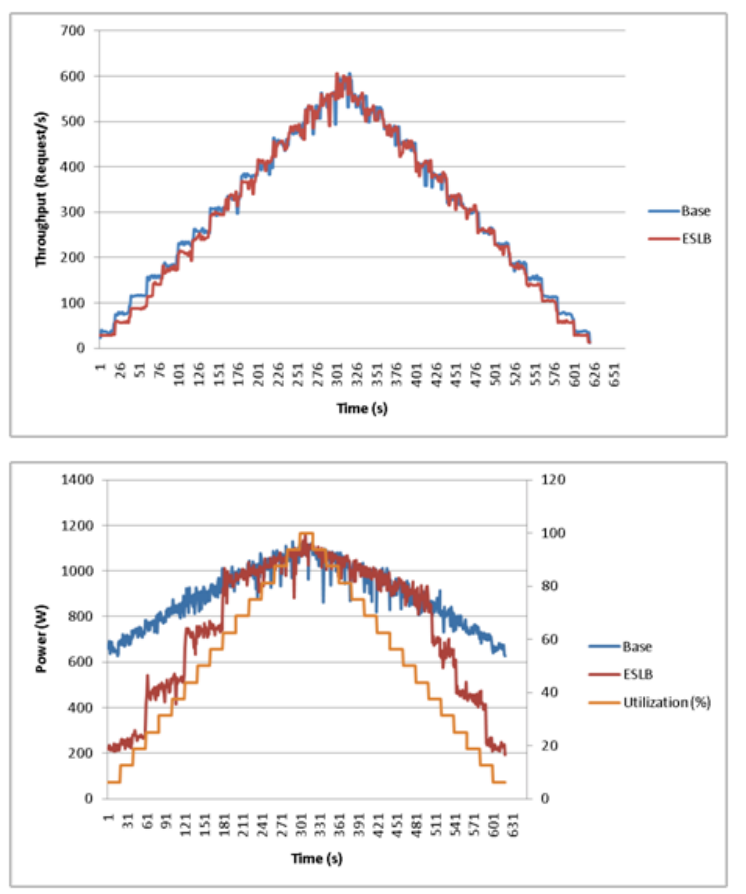

Figure 10. (a) Throughput, (b) power comparisons between the base system and the ESLB scheme when the load gradually changes

gained with a smarter load direction scheme at the global level.

Our technique can be adapted to cope with transients in the load by making the FEC(s) keep track of the rate of load changes and adjust the thresholds used by the smart load balancers accordingly. The FEC can also sense the load growth rate and adjust the number of servers that are activated simultaneously - the benefit of doing this was seen earlier in Section 5. Last but not the least, a number of servers can be kept on hot standby to cope with sudden load surges, as discussed earlier.

\section{Acknowledgements}

This work is supported in part by NYSERDA under agreement number 10946 and by the NSF awards, numbers 0958501 and 1040666.

\section{REFERENCES}

[1] Jonathan G. Koomey, "Estimating Total Power Consumption By Servers in the U.S. and the World", Analytics Press. Feb. 2007. Also available at: http://enterprise.amd.com/us-en/AMD-

Business/Technology-Home/Power-Management.aspx.

[2] Luiz André Barroso and Urs Hölzle, "The Datacenter as a Computer: An Introduction to the Design of
Warehouse-Scale Machines", Morgan-Claypool Publishers, 2009 (ISBN No. 9781598295566).

[3] J. Hamilton, "Where Does the Power Go In A Data Center?", keynote presentation at SIGMETRICS/Performance 2009.

[4] Bowden, T. and Bauer, B., "The /proc Filesystem", Linux Kernel Documentation, Oct 7, 1999.

[5] IBM Corporation, IBM Tivoli Usage Accounting Manager V7.1 Handbook, IBM Redbook, March 2008.

[6] IBM Corporation, Value Proposition for IBM Systems Director: Challenges of Operational Management for Enterprise Server Installations, IBM ITG Group, Mangement Brief (34 pages), Nov. 2008.

[7] Rusu, C., Ferreira, A., Scordino, C., Watson, A., Melhem. R. and Mosse, D., Energy-Efficient Real-Time Heterogeneous Server Clusters, Real-Time and Embedded Technology and Applications Symposium, 2006. Proceedings of the 12th IEEE, April 24, 2006.

[8] Krioukov, A., Mohan, P., Alspaugh, S., Keys, L., Culler, D., and Katz, R., "NapSAC: Design and Implementation of a Power-Proportional Web Cluster", Green Networking 2010, August 30, 2010

[9] Lang, W., Patel, J. M. and Naughton, J. F., "On energy management, load balancing and replication" in SIGMOD Rec. 38, 4 (June 2010), 35-42

[10] Alspaugh, S., Keys, L., Krioukov, A., "Power Proportional Cluster", 2009

[11] Chun, B. G., Iannaccone, G., Iannaccone, G., Katz, R., Lee, G., and Niccolini, L., "An energy case for hybrid datacenters", in ACM SIGOPS Operating Systems Review 44 (1): 76-80, 2010.

[12] Meisner, D., B. T. Gold, and T. F. Wenisch. 2009. PowerNap: Eliminating server idle power. $A C M$ SIGPLAN Notices 44 (3): 205-16.

[13] Pinheiro, E., Bianchini, R., Carrera, E. V., and Heath, T., "Dynamic cluster reconfiguration for power and performance", in Compilers and operating systems for low power, edited by L. Benini, M. Kandemir, and J. Ramanujam, Kluwer Academic Publishers, 2003, pp. 7593.

[14] Akshat Verma, Puneet Ahuja, Anindya Neogi "pMapper: Power and Migration Cost Aware Application Placement in Virtualized Systems", In Middleware '08: Proceedings of the 9th ACM/IFIP/USENIX International Conference on Middleware (2008), pp. 243-264.

[15] "Intel 64 and IA-32 Architectures Software Developer's Manual”, Volume 3B, January 2011.

[16] Dell 610 Poweredge server product literature, 2010.

[17] Apache "ab Benchmark" web pages at: http://httpd.apache.org/docs/2.0/programs/ab.html, 2011. 